\title{
Collision Detection Using a Flexible Link Manipulator : A Feasibility Study
}

\author{
Stewart J. Moorehead and David Wang \\ Department of Electrical and Computer Engineering \\ University of Waterloo \\ Waterloo, Ontario N2L 3G1
}

\begin{abstract}
A simple collision detection method for a flexible link manipulator is proposed. The method only requires two strain measurements to determine the position of a collision with the flexible link. Equations which relate the force and position of contact to the strain at two points in the flexible link are derived assuming the link is at static equilibrium. Since the flexible robot is actually in motion, one needs to examine whether these relationships are accurate enough in a dynamic scenario to be useful Thus the expressions are verified on a robot through collision tests, and used to successfully locate an encountered object so that a path can be planned around it.
\end{abstract}

\section{Introduction}

As robotic technology matures, the requirements for speed and efficiency increase. Up until now the increase in productivity provided by robots has been sufficient, but to further increase this productivity, novel ideas are necessary. Currently one of the limiting factors of robots is the need to work in a highly structured environment. This restriction is required to prevent collision with objects in the robot's workspace. In particular, this often precludes human operators from being inside the workspace. In some situations it may be helpful, or necessary, for a human operator to be in close proximity to the robot.

There are two main ways to give a robot the knowledge it needs to operate in an unstructured environment. The first method is to use a vision system. The shortcoming of this solution is that it is often difficult to guarantee that every possible point of collision will be in the field of view of the camera, particularly in an unstructured and dynamic environment. The second method is to give the robot a sense of touch. In $[2,3,4]$ a proximity sensing skin is developed which covers the entire robot arm. The systems allow the robot to know when any point of its arm is near an object. The major drawback of this approach is that it requires large amounts of processing power and is fairly complicated. Another method to give the robot a sense of touch is through the use of flexible links with strain gauges. In $[8,9]$ a system for measuring the position of contact is developed which uses force and moment information provided by a force/ torque sensor. In [5] a sensor system is proposed for a mobile robot which is inspired by the antennae of an insect. The sensor is a flexible beam which is actuated at one end. When the antenna comes into contact with an object the actuator continues to move through an angle so that a contact force is generated. By measuring the torque at a point on the beam as well as the angle the actuator moved after the collision was detected, Kaneko is able to determine the position of contact on the antenna. As in this paper, these methods look at the static case. Dynamic effects are taken into account in [10] which measures the frequency of the fundamental mode of vibration to determine the collision point. However they require the addition of a mass at the end of the beam to be able to uniquely determine the contact position from the frequency measurement.

This paper presents a preliminary feasibility study to provide a robot arm with a sense of touch. The method uses a flexible link robot, and two strain gauges to determine the magnitude and position of an externally applied force. A simple static model of the beam is proposed, from which equations relating the magnitude and position of an applied force to the strain in the beam, are derived. This is followed by a brief discussion of how the equations were implemented to allow collision detection.

Since two strain gauges are necessary to control the first two modes of vibration in the flexible link for unconstrained movements, the collision detection method proposed in this paper does not require the addition of any sensors. A static model of the beam was chosen because in the future this method is to be extended to a robot where all the links are flexible, allowing the detection of a collision with any part of the robot. If a more complex model of the beam was used it may not be 
possible to extend it to the multilink case. Due to the assumption of static behavior, it is extremely important to verify experimentally that this approach is feasible. This paper shows data confirming that this technique is plausible.

It should be noted that the flexible nature of the links is essential to this methodology. The algorithm presented here would not work well for a typical rigid robot manipulator as it would be extremely difficult for a collision, especially with a human operator, to create a measurable strain. This safety hazard makes it impossible to allow humans inside the workspace of a rigid robot. By using the method presented in this paper and a flexible robot it will be possible to allow robots and humans to work in close proximity. Thus in addition to the usually accepted advantages of flexible link manipulators, e.g. speed, low cost and compliance, one can now add safety.

\section{Modeling}

In this section a basic model of the flexible link is developed. The goal of this model is to provide very simple relationships between the force and position of contact with the strain at specific points on the beam. The following model considers a beam which is in static equilibrium. There is one external force applied and its magnitude is small enough so that the deformation of the beam from its rest position is small. Furthermore only forces in the $y$ direction are considered in this preliminary investigation.

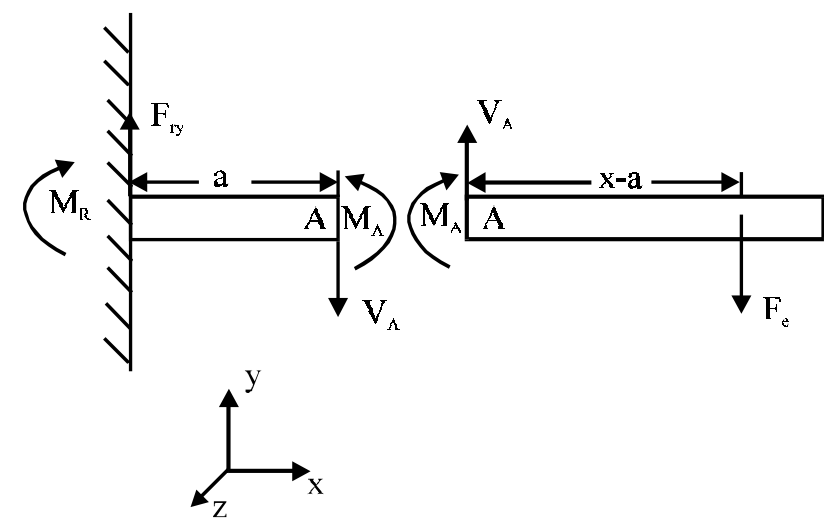

Figure 1-Cantilever Beam

As shown in Figure 1, the flexible link is modeled as a cantilever beam. The environment exerts a force $F_{e}$ on the beam at a position $x$. At point $\mathbf{A}$ a strain gauge is mounted, and in Figure 1 the beam has been cut here to show the moment and shear force present at the strain gauge. Since only applied forces in the $y$ direction are being considered, the following two conditions must be met for static equilibrium,

$$
\sum F_{y}=0 \quad \sum M_{z}=0
$$

Now equation (1) must hold for each of the two pieces in Figure 1. Taking the right hand piece it is possible to write,

$$
\sum F_{y}=0=F_{e}-V_{A} \Rightarrow V_{A}=F_{e}
$$

and

$$
\begin{aligned}
& \sum M_{a t_{-} A}=F_{e}(x-a)-M_{A}=0 \\
& M_{A}=F_{e}(x-a)
\end{aligned}
$$

From [6],

$$
M_{A}=\frac{\varepsilon_{a} E I}{c}
$$

where $\varepsilon_{a}$ is the strain at point $\mathbf{A}$ and $c$ is half the thickness of the beam. Thus by combining equations (3) and (4), an expression for the applied force in terms of the strain at point $\mathbf{A}$ is found.

$$
F_{e}=\frac{\varepsilon_{a} E I}{c(x-a)}
$$

If a second strain gauge is placed at a different position on the beam, $\mathbf{B}$, then a similar expression for the external force can be written in terms of $\varepsilon_{b}$. Finally by equating these two expressions for the force, it is possible to solve for the position of contact, $x$.

$$
x=\frac{\varepsilon_{a} b-\varepsilon_{b} a}{\varepsilon_{a}-\varepsilon_{b}}
$$

These expressions could be extended to account for the acceleration of the beam by using D'Alembert's Principle. This would mean that the summations in equation (1) would no longer be equal to zero, which would change equations (2) through (6). As well it would be necessary to know what side of the beam's center of mass $F_{e}$ is on.

Equations (5) and (6) are very simple expressions for the force and position of a collision between the flexible link and an external object. They will be used in the following sections to implement a collision detection system, and allow the robot to plot a simple trajectory around the object. 


\section{Implementation}

The experiments described below were implemented on a 5 bar robot as shown in Figure 2. The parallelogram structure of the robot is rigid, however the part of link 4 extending past link 1 is made of 1/4" square aluminum, and is $1 \mathrm{~m}$ long, making it very flexible.

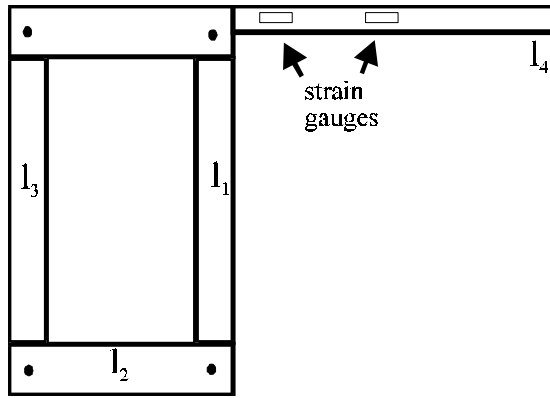

Figure 2- Five Bar Robot

Attached to this link are two sets of strain gauges so that the position of an external force can be determined. These strain gauges are situated at $1.5 \mathrm{~cm}$ and $21.6 \mathrm{~cm}$ from the point where link 1 and link 4 meet. The entire system, robot and strain gauges, is controlled and monitored by a single 486 computer. The digital controller uses a sample time of $5 \mathrm{~ms}$.

\subsection{Static Tests}

To determine the accuracy of equations (5) and (6) some static tests were performed on the robot of Figure 2. A known force was applied to the beam at various known locations, and strain measurements were taken. Equations (5) and (6) used these strain measurements to get the calculated values of force and position. The results of these tests, for two different forces, are shown in Figures 3 and 4.

Figure 3 shows the results of several tests. The $x$ 's represent the actual force applied and the +'s represent the force calculated using (5). The results are plotted versus the position on the beam where the force was applied with $1 \mathrm{~m}$ corresponding to the tip of the flexible link. It is quite clear that (5) does not yield an accurate value for the applied force. The error can in part be explained by the deformation of the beam due to the external force. Since the derivation of (5) required this to be small, as the deformation increases, (5) becomes less valid. Fortunately for application to the problem of collision detection the force is not strictly required. Equation (5) does however give an idea of the magnitude of the applied force, and can thus be used to indicate how much force is generated by a collision so that the suitability of a robot for human interaction can be determined.

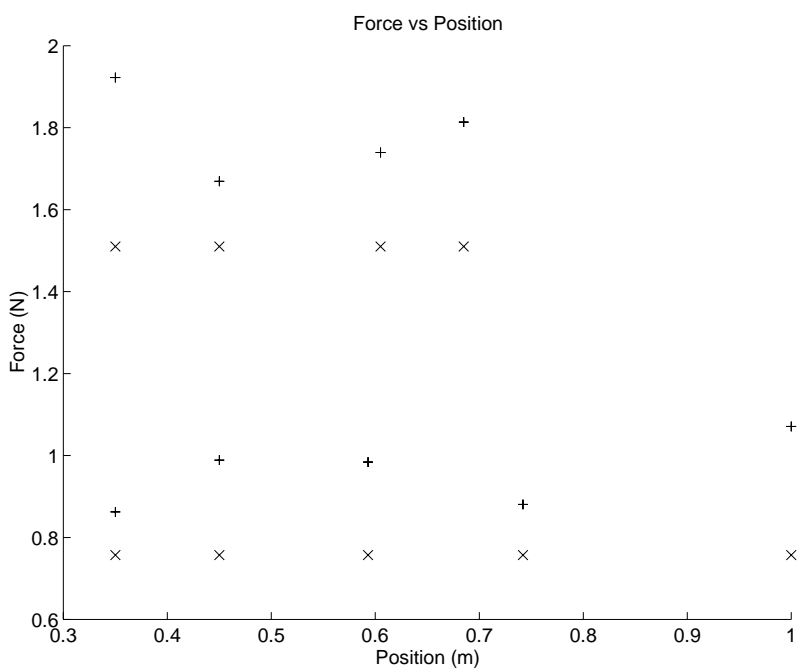

Figure 3

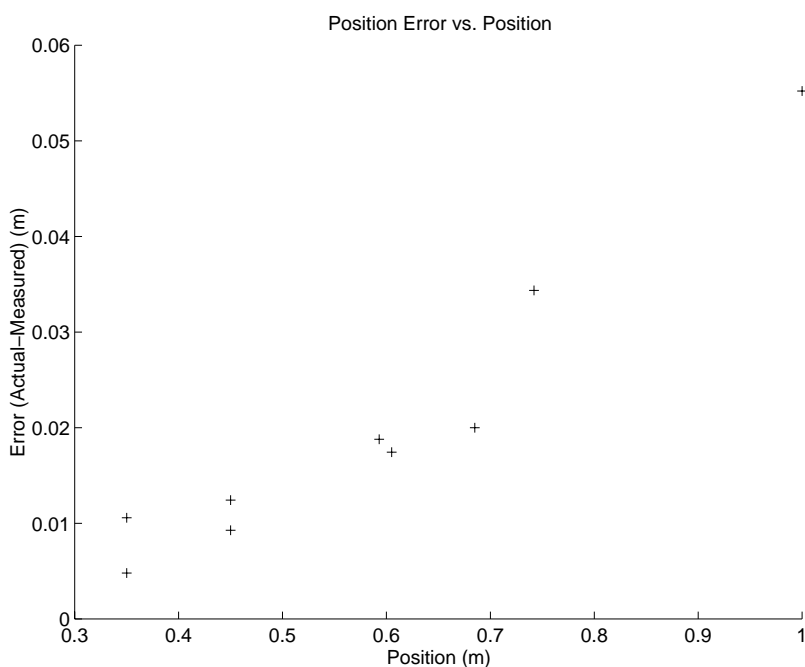

Figure 4

Figure 4 shows the error in calculated position (using (6)) versus the position of the applied force. These results show that it is possible to get an accurate measurement of the collision point. This is important so that a valid trajectory can be made to go around the detected object. As expected, the error gets larger as the applied force gets closer to the tip.

\subsection{Collision Detection}

When the flexible link is moved through a motion without hitting an object, it will vibrate. These vibrations will cause strain in the beam which will be 
measured by the strain gauges. If the readings of the strain gauges are applied to (5) and (6) a position and force will be calculated. While no collision has in fact occurred, the vibrations have caused a deformation in the beam. The calculated position and force represent an external force which would produce the same deformation in the static case.

Since (5) and (6) cannot distinguish between strains caused by a collision and those caused by beam vibration some other criterion must be used to determine when a collision has occurred. In [7], Richter et. al. use a flexible robot for force control. To detect a collision they simply wait until the measured force exceeds a prespecified threshold. In this paper, it was decided to instead wait for the first time derivative of the strain to exceed a prespecified threshold. Figure 7 shows the value of the first derivative of strain vs. time. The robot collides with the object just after 500 sample periods. As the graph shows, there is a significant spike in the strain derivative during a collision.

\section{Dynamic Tests}

The equations of section 2 are static where as the robot is a dynamic system. To verify that the static results are valid in this dynamic situation the following experiment was conducted.

To demonstrate the feasibility of using (6) for locating the point of collision between the robot and an object, a simple experiment was set up. The 5 bar robot described in section 3 was commanded to move the base motor from 0 to -1 rad. Each joint on the robot was controlled with a rigid body PID controller (i.e. the controller was designed assuming no link flexibility). An object had been placed in the path of the robot so that a collision would occur.

When the collision was detected using the method described in section 3.2 the following algorithm was executed to allow the robot to go around it.

1. stop the arm

2. use equation (6) to get the position of the collision

3. assume that the object occupies an area enclosed by a semi-circle with radius $5 \mathrm{~cm}$ centered at the point of contact

4. back the arm away from the object by setting the reference point of the base controller to $0.1 \mathrm{rad}$ more than the current location

5. transform the position of contact to the robot's base coordinate frame
6. use the robot's inverse kinematics to calculate the side motor angles required to let the tip of link 4 pass by the object

7. move the side motors to the new positions

8. resume the original base movement

9. when the tip of link 4 is past the object, restore the side motors to the original values

Since the current experimental apparatus is not able to detect longitudinal deformations of the flexible link, the robot is unable to detect collisions during this restoring motion (step 9). For the system to be truly operable in an unstructured environment sensors will have to be installed to detect these longitudinal deformations.

In Figure 5, the base and left motor angles for a collision test are presented. The base travels from 0 to -1 rad while the left motor goes from approximately 1.5 to 2.0 and back to $1.5 \mathrm{rad}$. The time of collision is indicated by the vertical line on the graph. It is possible to see in this figure how the robot backs off from the object and then the side motors are moved so that the object may be avoided.

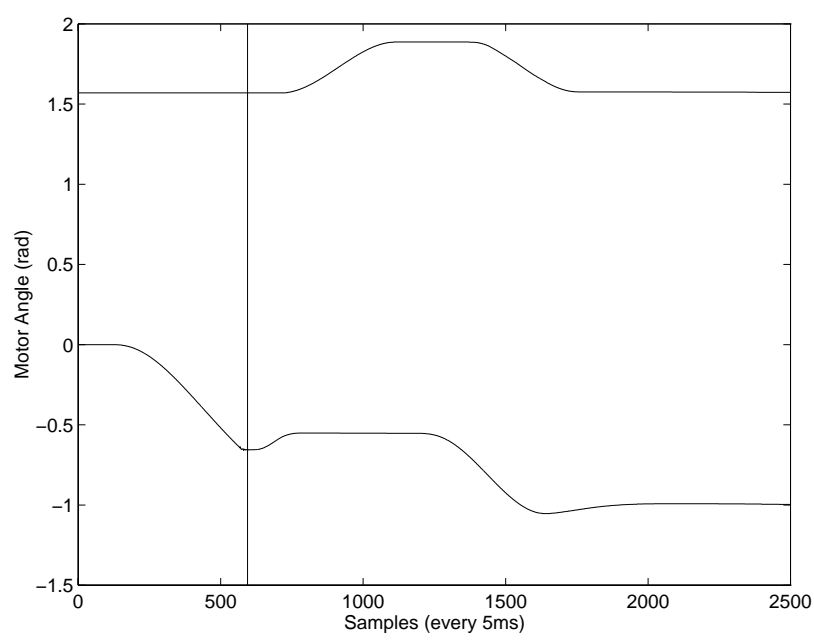

Figure 5-Base and Left Motor Angles

In Figure 6, the result of equation (6) is presented for a dynamic trial. While the flexible beam is moving in an unconstrained environment, it will vibrate. These vibrations deform the beam from its rest position. If only the first mode of vibration is considered, it is possible to construct an external force which will generate the same beam shape. The position of this force is what equation (6) calculates during unconstrained motion, and is shown on Figure 6. However around the 600th sample the position value settles down briefly. Comparing this with Figure 5, it is possible to see that this is where the collision has occurred. Since the time of contact with the object is fairly short the time axis of Figure 6 has been 
expanded to show more clearly the time of impact. Notice that while the beam is in contact with the object, the position estimate is not a constant as would be expected. There are several factors which cause the oscillations present in the position reading during contact. Largely, however, it is due to the beam bouncing off of the contacted object. To overcome these errors, the position is calculated 20 times and then averaged.

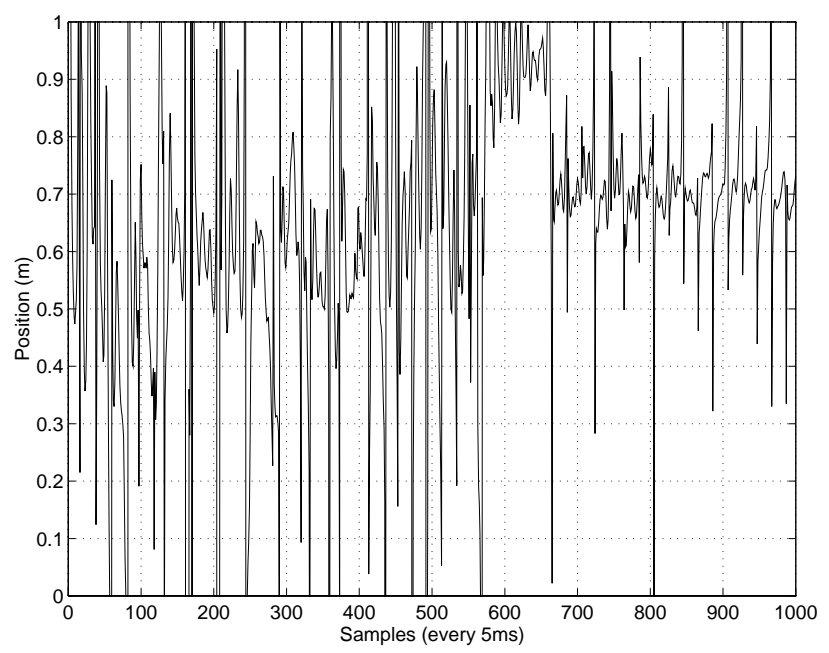

Figure 6-Collision Position

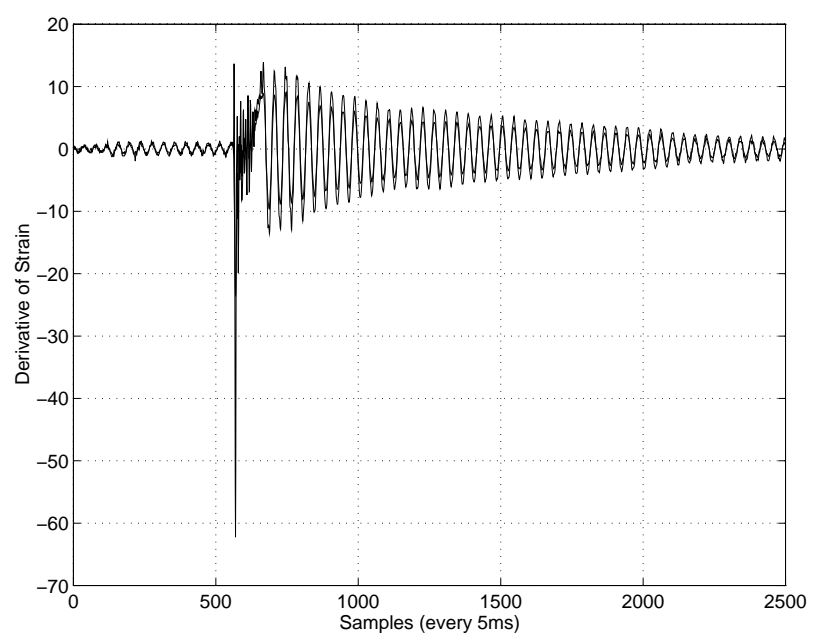

Figure 7-Derivative of Strain

Figure 7 shows the first time derivative of the strain as measured by the first strain gauge. The large negative spike just after 500 sample periods occurs when the robot hits an object. The oscillations before and after this point represent vibrations in the beam due to its flexibility. This graph clearly shows that it is possible to determine the time of collision by looking at the first time derivative of the strain, as was proposed in section 3.2.

\section{Conclusions}

This paper presented an effective yet simple method to allow a robot arm to safely interact in a some what unstructured environment. Two very simple expressions were derived which allowed the magnitude and position of an external force to be calculated using only two distinct strain measurements on the link. While the model used to derive these equations was very simplistic, the results were verified by performing static and dynamic tests on a physical system. While some error was present, the results were accurate enough to sufficiently locate an object so that a path could be planned around it. This was shown in section 4 .

The results presented in this paper were intended to demonstrate a proof of concept only. Clearly much more work is necessary for this procedure to be used in a truly unstructured environment. While the force measurement is accurate enough for the application presented here, it must be made more accurate if this concept is to be extended to force control. Another important change involves the consideration of forces of arbitrary direction. Currently only forces in the $y$ direction (see Figure 2) are considered. It is fairly easy to extend these concepts to measuring forces in the $x$ and $z$ directions. Forces in the $z$ direction can be measured in the same manner as those in the $y$ direction by simply using two strain gauges on the $x-y$ face of the beam. Forces in the $x$ direction are more difficult to measure and require some manner of measuring the axial stress in the beam.

Currently a very simple algorithm is used to navigate past objects in the workspace. With a truly unstructured environment a more sophisticated algorithm needs to be used for obstacle avoidance. As well, the current robot can only detect collisions which occur on link 4. It is possible, of course, for other parts of the robot to come in contact with an object. Conceptually it is very simple to imagine adding strain gauges to all links, thus allowing the robot to detect collisions on any part of its arm. However since a rigid link would generate a large amount of force before the collision was detected, it would be necessary to have a robot in which all links were flexible. The simplicity of the results in this paper should ease the extension of these concepts to a multilink flexible robot.

\section{References}

[1] Beer F., E.R. Johnston, Mechanics of Materials, McGraw-Hill, 1981. 
[2] Cheung E., V. Lumelsky, Development of Sensitive Skin for a 3D Robot Arm Operating in an Uncertain Environment, IEEE Int. Conf. on Robotics and Automation, p. 1056-1061, 1989.

[3] Cheung E., V. Lumelsky, Motion Planning for Robot Arm Manipulators with Proximity Sensing, IEEE Int. Conf. on Robotics and Automation, p. 740-745, 1988.

[4] Feddema J.T., J.L. Novak, Whole Arm Obstacle Avoidance for Teleoperated Robots, IEEE Int. Conf. on Robotics and Automation, p. 3303-3309, 1994.

[5] Kaneko M., Active Antenna, IEEE Int. Conf. on Robotics and Automation, p. 2665-2671, 1994.

[6] Popov E.P, Mechanics of Materials, Prentice-Hall, 1976.

[7] Richter K., F. Pfeiffer, A Flexible Link Manipulator as a Force Measuring and Controlling Unit, IEEE Int. Conf. on Robotics and Automation, p. 1214-1219, 1991.

[8] Tsujimura T., T. Yabata, A Tactile Sensing Method Employing Force/Torque Information Through Insensitive Probes, IEEE Int. Conf. on Robotics and Automation, p. 1315-1320, 1992.

[9] Tsujimura T., T. Yabata, Object Detection by Tactile Sensing Method Employing Force/Torque Information, IEEE Trans. on Robotics and Automation, Vol. 5, No 4, 1988.

[10] Ueno N., M. Kaneko, Dynamic Active Antenna, IEEE Int. Conf. on Robotics and Automation, p. 17841790, 1994. 